\title{
LA ALFABETIZACIÓN EN INFORMACIÓN COMO DIMENSIÓN DE UN NUEVO MODELO EDUCATIVO: LA INNOVACIÓN DOCENTE DESDE LA DOCUMENTACIÓN Y LOS CRAI ${ }^{1}$
}

\author{
(INFORMATION LITERACY AS EDUCATIVE DIMENSION OF THE LRC: CONVERGENCE \\ BETWEEN INFORMATION SERVICES AND TEACHING INNOVATION)
}

\author{
Miguel Ángel Marzal \\ Universidad Carlos III de Madrid (España)
}

\section{RESUMEN}

Desde el análisis de las repercusiones educativas del Ciberespacio se analiza, como uno de sus elementos, la relevancia de las competencias en Educación y su proyección social formativa, base fundamental para el desarrollo de la alfabetización en información, que es considerada desde su perspectiva conceptual, normativa y metodológica. La dimensión educativa de la alfabetización en información se contempla como una competencia en la evaluación de contenidos digitales, con modelos propios, porque debe tener como efectos en el educando por comprensión eficaz y generación de conocimiento desde la lectura digital, escritura hipermedia y edición. El impacto de la alfabetización en información se hace eficiente por recursos digitales idóneos (objetos de aprendizaje, blogs), en el escenario lógico que es el CRAI, cuyos instrumentos necesarios, proyecto y tendencias formativas se abordan.

Palabras clave: alfabetización en información, CRAI, competencias, evaluación de contenidos digitales.

\begin{abstract}
One of the most crucial aspects in the analysis of the educational impact of Cyberspace is the relevance of competencies in education and their formative social outreach. It is the fundamental base for the development of information literacy, which is approached in this paper from its conceptual, regulatory and methodological perspective. The educational dimension of information literacy is considered here as a competency in the evaluation of digital learning resources provided with models of its own that will be able to allow students to efficiently understand and generate knowledge from digital reading, hypermedia writing and editing. The impact of information literacy is, then, proved to be efficient by means of appropriate digital resources (learning objects and blogs, among others) in their natural setting, the CRAI (Learning Resources Centers), whose instruments, project and formative trends are also tackled in this paper.
\end{abstract}


Keywords: information literacy, LRC, competences, digital content evaluation.

Los inicios aplicativos de los principios programáticos del Espacio Europeo de Educación Superior (en adelante EEES) en España han implicado, no sólo una reflexión sobre la innovación docente para un nuevo modelo de Educación, sino un esfuerzo de aplicación planificada de acciones educativas experimentales preliminares, que incorporaban como instrumento el entorno tecnológico. El Ciberespacio se ha evidenciado como el espacio educativo de primera magnitud, que sin duda debe ser incorporado por la universidad, empeño en el que las Tecnologías de la Información y Comunicación (en adelante TIC) y los instrumentos de gestión de contenidos, procedentes de la Documentación, deben comportarse como herramientas necesarias en la consecución de ese objetivo, en tanto que paradigma del nuevo modelo educativo. El EEES, con todas sus dificultades, debe aspirar no sólo a ser un mecanismo de reforma educativa, sino el soporte de un nuevo modelo de Educación que sostenga en la Unión Europea la Educación para la Sociedad del Conocimiento. El EEES debe servir de plataforma para garantizar la sinergia necesaria en un escenario nuevo y diferente en los modos de conocer, en las herramientas para enseñar y aprender en una escuela distinta, en los objetivos que para la formación permanente de sus ciudadanos la Unión Europea pergeñó en los planes de e-Europe 2002 y 2005.

No cabe duda de que en la enunciación del método aplicativo de Educación apuntado en el EEES, su seguimiento evaluativo y la formulación modélica de un nuevo sistema educativo, es imprescindible la confluencia de las distintas perspectivas epistemológicas, metodológicas e instrumentales de aquellas Ciencias que puedan ser útiles en el empeño de una Educación para la Sociedad del Conocimiento, entre ellas indefectiblemente la Documentación. En efecto, la Documentación puede ser particularmente útil en la eficacia de la convergencia de tres importantes estímulos: la eficiencia en el nuevo modelo de Educación de la innovación docente por la incorporación "alfabetizadora" de las TIC; la incorporación "organizada, comprensiva y sistemática” de la información del Ciberespacio, como conocimiento en las aulas extendidas en la red; el decidido desarrollo en los instrumentos de gestión de contenidos digitales, actuales y futuribles, de su capacidad y dimensión instructivas.

En este esfuerzo convergente, el concurso de la Documentación se muestra particularmente atractivo en cinco planos de acción: la transformación de las competencias en el "nervio y sangre" de los principios educativos en el aula extendida, por ser fundamento del "pensamiento complejo"; la primacía de los objetivos de 
Educación sobre las estrategias de enseñanza y aprendizaje en el sistema competencial del nuevo modelo educativo; la inoculación en el modelo educativo de una "cultura de la evaluación”, no referida al mecanismo propedéutico de ascenso curricular del educando en el sistema escolar, sino a una evaluación del impacto de las acciones y políticas TIC en la eficiencia para la consecución de más y mejor conocimiento y formación en el educando; la integración curricular de la alfabetización en información, en tanto que competencia fundamental de lectura por captación eficaz e inteligente de recursos web para articular y estructurar un nuevo conocimiento, así como competencia en escritura, conforme a una "gramática" web interoperable, compartida, asociativa y compatible en red; la transformación de las bibliotecas universitarias en CRAI, donde su colección digital educativa, integrada en una parte relevante por los objetos de aprendizaje, sea fundamento de las habilidades y destrezas necesarias en las competencias para el aprendizaje permanente de la Educación para la Sociedad del Conocimiento. Una reflexión expositiva de estas aportaciones de la Documentación es la razón de este artículo.

\section{LAS COMPETENCIAS PARA EL APRENDIZAJE PERMANENTE EN EL AULA EXTENDIDA}

En un contexto educativo las competencias deben ser consideradas como prólogo de las destrezas manipulativas y las habilidades intelectivas de que el educando debe adquirir y desarrollar para su pleno progreso personal, laboral e intelectual. Esta secuencia, en la que las habilidades y destrezas no sólo se diferencian conceptualmente sino que se ordenan con antelación a las competencias en el proceso educativo, es muy importante asimilarla en la nueva Educación.

Las competencias, en efecto, comienzan su andadura conceptual en el siglo XX referidas a la consecución de capacidades en un proceso productivo, aun cuando los inicios de Internet remitieron las competencias hacia una acepción más conductista, en cuanto que conjunto de destrezas y habilidades estructuradas para la consecución de objetivos propuestos. Las competencias se reflejaban en aptitudes para el ejercicio de una profesión, la resolución de problemas y la organización del trabajo, por lo que Punk distinguió competencias técnicas, metodológicas y sociales² ${ }^{2}$. Sin embargo, en los comienzos del siglo XXI, las competencias pasan a combinarse decididamente con la información: Le Boterf las entiende como una construcción, resultado de la combinación de conocimientos, redes de relación y saber hacer ${ }^{3}$. La razón de este cambio conceptual cualitativo no ofrece dudas, por cuanto paralelamente comenzaba a emerger la economía del conocimiento, en la que uno de sus principios descansaba en optimizar no tanto la producción, sino la calidad de esa producción, 
en íntima relación con una elevada cualificación profesional de la mano de obra por adecuación al acelerado ritmo de la innovación científico-tecnológica y los cambios en la estructura y organización del trabajo. Las competencias, así, alcanzaban una indudable dimensión educativa: una educación en competencias debe objetivarse en resultados, que son los productos del aprendizaje (conocimientos, habilidades, destrezas, aptitudes y actitudes).

Las competencias se convertían en actor del nuevo modelo educativo ${ }^{4}$, a las que se encomendaban desarrollar en el educando estímulos a partir de destrezas y habilidades estructuradas: potencialidades personales, como conocimiento, motivación, actitud, habilidades cognitivas y prácticas; potencialidades funcionales, como capacidades de programación, gestión, ejecución y evaluación, aptitudes y destrezas en el desempeño de tareas; potencialidades contextuales, como actitudes de superación, renovación, actualización, cultura sistémica de organización y cooperativismo. En esta dimensión didáctica, las competencias se clasificaban en competencias básicas, genéricas y específicas en el proceso educativo. Desde un punto de vista conceptual las competencias lograban un rango de objetivo educativo de primera magnitud, tal como quedaron definidas por Lévy-Leboyer (2003), como "conjunto de conductas organizadas en el seno de una estructura mental, también organizada y relativamente estable y movilizable cuando es preciso ${ }^{5 "}$. Desde un punto de vista programático, la Unión Europea publicaba el Proyecto Tunning Educational structures in Europe, cuyo objetivo era considerar los títulos universitarios en términos de resultados de aprendizaje y de competencias, tanto genéricas (instrumentales, interpersonales y sistemáticas), como específicas de cada área de conocimiento.

La centralidad de las competencias transforma el modelo educativo: las estrategias didácticas de aprendizaje tendrán por objeto la autonomía del educando, absolutamente implicado y protagonista en su proceso formativo ${ }^{6}$, mientras que las estrategias didácticas de enseñanza se orientarán a formar al educando en habilidades, destrezas y competencias, siguiendo un orden cognitivo creciente. Este marco educativo debe tener por espacio natural el aula extendida, un espacio virtual de acogida para comunidades digitales educativas, organizadas en torno a un tópico educativo de interés (relegando la estructura curricular clásica de "materia") conforme a un diseño instructivo de adquisición de competencias proyectadas complementariamente en unidades de aprendizaje (unidades didácticas) y unidades de competencia (fundadas sobre los objetos de aprendizaje).

El aula extendida debe pivotar sobre los elementos formativos de las TIC más relevantes en una comunidad digital educativa, a saber: la interactividad de los 


\title{
LA ALFABETIZACIÓN EN INFORMACIÓN COMO DIMENSIÓN DE UN NUEVO MODELO EDUCATIVO: LA INNOVACIÓN DOCENTE DESDE LA DOCUMENTACIÓN Y LOS CRAI ${ }^{1}$
}

\author{
(INFORMATION LITERACY AS EDUCATIVE DIMENSION OF THE LRC: CONVERGENCE \\ BETWEEN INFORMATION SERVICES AND TEACHING INNOVATION)
}

\author{
Miguel Ángel Marzal \\ Universidad Carlos III de Madrid (España)
}

\section{RESUMEN}

Desde el análisis de las repercusiones educativas del Ciberespacio se analiza, como uno de sus elementos, la relevancia de las competencias en Educación y su proyección social formativa, base fundamental para el desarrollo de la alfabetización en información, que es considerada desde su perspectiva conceptual, normativa y metodológica. La dimensión educativa de la alfabetización en información se contempla como una competencia en la evaluación de contenidos digitales, con modelos propios, porque debe tener como efectos en el educando por comprensión eficaz y generación de conocimiento desde la lectura digital, escritura hipermedia y edición. El impacto de la alfabetización en información se hace eficiente por recursos digitales idóneos (objetos de aprendizaje, blogs), en el escenario lógico que es el CRAI, cuyos instrumentos necesarios, proyecto y tendencias formativas se abordan.

Palabras clave: alfabetización en información, CRAI, competencias, evaluación de contenidos digitales.

\begin{abstract}
One of the most crucial aspects in the analysis of the educational impact of Cyberspace is the relevance of competencies in education and their formative social outreach. It is the fundamental base for the development of information literacy, which is approached in this paper from its conceptual, regulatory and methodological perspective. The educational dimension of information literacy is considered here as a competency in the evaluation of digital learning resources provided with models of its own that will be able to allow students to efficiently understand and generate knowledge from digital reading, hypermedia writing and editing. The impact of information literacy is, then, proved to be efficient by means of appropriate digital resources (learning objects and blogs, among others) in their natural setting, the CRAI (Learning Resources Centers), whose instruments, project and formative trends are also tackled in this paper.
\end{abstract}


Keywords: information literacy, LRC, competences, digital content evaluation.

Los inicios aplicativos de los principios programáticos del Espacio Europeo de Educación Superior (en adelante EEES) en España han implicado, no sólo una reflexión sobre la innovación docente para un nuevo modelo de Educación, sino un esfuerzo de aplicación planificada de acciones educativas experimentales preliminares, que incorporaban como instrumento el entorno tecnológico. El Ciberespacio se ha evidenciado como el espacio educativo de primera magnitud, que sin duda debe ser incorporado por la universidad, empeño en el que las Tecnologías de la Información y Comunicación (en adelante TIC) y los instrumentos de gestión de contenidos, procedentes de la Documentación, deben comportarse como herramientas necesarias en la consecución de ese objetivo, en tanto que paradigma del nuevo modelo educativo. El EEES, con todas sus dificultades, debe aspirar no sólo a ser un mecanismo de reforma educativa, sino el soporte de un nuevo modelo de Educación que sostenga en la Unión Europea la Educación para la Sociedad del Conocimiento. El EEES debe servir de plataforma para garantizar la sinergia necesaria en un escenario nuevo y diferente en los modos de conocer, en las herramientas para enseñar y aprender en una escuela distinta, en los objetivos que para la formación permanente de sus ciudadanos la Unión Europea pergeñó en los planes de e-Europe 2002 y 2005.

No cabe duda de que en la enunciación del método aplicativo de Educación apuntado en el EEES, su seguimiento evaluativo y la formulación modélica de un nuevo sistema educativo, es imprescindible la confluencia de las distintas perspectivas epistemológicas, metodológicas e instrumentales de aquellas Ciencias que puedan ser útiles en el empeño de una Educación para la Sociedad del Conocimiento, entre ellas indefectiblemente la Documentación. En efecto, la Documentación puede ser particularmente útil en la eficacia de la convergencia de tres importantes estímulos: la eficiencia en el nuevo modelo de Educación de la innovación docente por la incorporación "alfabetizadora" de las TIC; la incorporación "organizada, comprensiva y sistemática” de la información del Ciberespacio, como conocimiento en las aulas extendidas en la red; el decidido desarrollo en los instrumentos de gestión de contenidos digitales, actuales y futuribles, de su capacidad y dimensión instructivas.

En este esfuerzo convergente, el concurso de la Documentación se muestra particularmente atractivo en cinco planos de acción: la transformación de las competencias en el "nervio y sangre" de los principios educativos en el aula extendida, por ser fundamento del "pensamiento complejo"; la primacía de los objetivos de 
Educación sobre las estrategias de enseñanza y aprendizaje en el sistema competencial del nuevo modelo educativo; la inoculación en el modelo educativo de una "cultura de la evaluación”, no referida al mecanismo propedéutico de ascenso curricular del educando en el sistema escolar, sino a una evaluación del impacto de las acciones y políticas TIC en la eficiencia para la consecución de más y mejor conocimiento y formación en el educando; la integración curricular de la alfabetización en información, en tanto que competencia fundamental de lectura por captación eficaz e inteligente de recursos web para articular y estructurar un nuevo conocimiento, así como competencia en escritura, conforme a una "gramática" web interoperable, compartida, asociativa y compatible en red; la transformación de las bibliotecas universitarias en CRAI, donde su colección digital educativa, integrada en una parte relevante por los objetos de aprendizaje, sea fundamento de las habilidades y destrezas necesarias en las competencias para el aprendizaje permanente de la Educación para la Sociedad del Conocimiento. Una reflexión expositiva de estas aportaciones de la Documentación es la razón de este artículo.

\section{LAS COMPETENCIAS PARA EL APRENDIZAJE PERMANENTE EN EL AULA EXTENDIDA}

En un contexto educativo las competencias deben ser consideradas como prólogo de las destrezas manipulativas y las habilidades intelectivas de que el educando debe adquirir y desarrollar para su pleno progreso personal, laboral e intelectual. Esta secuencia, en la que las habilidades y destrezas no sólo se diferencian conceptualmente sino que se ordenan con antelación a las competencias en el proceso educativo, es muy importante asimilarla en la nueva Educación.

Las competencias, en efecto, comienzan su andadura conceptual en el siglo XX referidas a la consecución de capacidades en un proceso productivo, aun cuando los inicios de Internet remitieron las competencias hacia una acepción más conductista, en cuanto que conjunto de destrezas y habilidades estructuradas para la consecución de objetivos propuestos. Las competencias se reflejaban en aptitudes para el ejercicio de una profesión, la resolución de problemas y la organización del trabajo, por lo que Punk distinguió competencias técnicas, metodológicas y sociales² ${ }^{2}$. Sin embargo, en los comienzos del siglo XXI, las competencias pasan a combinarse decididamente con la información: Le Boterf las entiende como una construcción, resultado de la combinación de conocimientos, redes de relación y saber hacer ${ }^{3}$. La razón de este cambio conceptual cualitativo no ofrece dudas, por cuanto paralelamente comenzaba a emerger la economía del conocimiento, en la que uno de sus principios descansaba en optimizar no tanto la producción, sino la calidad de esa producción, 
en íntima relación con una elevada cualificación profesional de la mano de obra por adecuación al acelerado ritmo de la innovación científico-tecnológica y los cambios en la estructura y organización del trabajo. Las competencias, así, alcanzaban una indudable dimensión educativa: una educación en competencias debe objetivarse en resultados, que son los productos del aprendizaje (conocimientos, habilidades, destrezas, aptitudes y actitudes).

Las competencias se convertían en actor del nuevo modelo educativo ${ }^{4}$, a las que se encomendaban desarrollar en el educando estímulos a partir de destrezas y habilidades estructuradas: potencialidades personales, como conocimiento, motivación, actitud, habilidades cognitivas y prácticas; potencialidades funcionales, como capacidades de programación, gestión, ejecución y evaluación, aptitudes y destrezas en el desempeño de tareas; potencialidades contextuales, como actitudes de superación, renovación, actualización, cultura sistémica de organización y cooperativismo. En esta dimensión didáctica, las competencias se clasificaban en competencias básicas, genéricas y específicas en el proceso educativo. Desde un punto de vista conceptual las competencias lograban un rango de objetivo educativo de primera magnitud, tal como quedaron definidas por Lévy-Leboyer (2003), como "conjunto de conductas organizadas en el seno de una estructura mental, también organizada y relativamente estable y movilizable cuando es preciso ${ }^{5 "}$. Desde un punto de vista programático, la Unión Europea publicaba el Proyecto Tunning Educational structures in Europe, cuyo objetivo era considerar los títulos universitarios en términos de resultados de aprendizaje y de competencias, tanto genéricas (instrumentales, interpersonales y sistemáticas), como específicas de cada área de conocimiento.

La centralidad de las competencias transforma el modelo educativo: las estrategias didácticas de aprendizaje tendrán por objeto la autonomía del educando, absolutamente implicado y protagonista en su proceso formativo ${ }^{6}$, mientras que las estrategias didácticas de enseñanza se orientarán a formar al educando en habilidades, destrezas y competencias, siguiendo un orden cognitivo creciente. Este marco educativo debe tener por espacio natural el aula extendida, un espacio virtual de acogida para comunidades digitales educativas, organizadas en torno a un tópico educativo de interés (relegando la estructura curricular clásica de "materia") conforme a un diseño instructivo de adquisición de competencias proyectadas complementariamente en unidades de aprendizaje (unidades didácticas) y unidades de competencia (fundadas sobre los objetos de aprendizaje).

El aula extendida debe pivotar sobre los elementos formativos de las TIC más relevantes en una comunidad digital educativa, a saber: la interactividad de los 
materiales didácticos virtuales, captados, organizados y también editados por los miembros, docentes y discentes de la comunidad (objetos de aprendizaje), una parte, si bien sustancial, de la ciberoteca, que debe auxiliar la comunidad, como colección digital de recursos educativos; la edición de documentos digitales colegiados, leídos, creados, sucesivamente transformados por los miembros de la comunidad, conforme a pautas, normas y cronograma establecidos, a partir de las posibilidades ofrecidas por los blogs y la tecnología wiki; la comunicación telemática a tiempo real y diferido, soporte para el aprendizaje colaborativo y cooperativo; las herramientas de conexión con archivos abiertos institucionales, para el acceso a documentos educativos digitales con un valor y precisión didácticos de primera magnitud, según los tópicos; un catálogo digital educativo, constituido por mapas conceptuales en el almacenamiento de recursos educativos virtuales, y tópic maps para su difusión, un instrumento idóneo para el aprendizaje significativo en el Ciberespacio.

El aula extendida, así concebida y diseñada para un modelo educativo competencial, no se identifica en modo alguno a un modelo de "escuela virtual". Muy al contrario, el aula extendida encuentra su verdadera razón educativa en un modelo de escuela presencial, esto es, un modelo que infiera necesariamente la convivencia y socialización de los educandos (modo inevitable para formar en "educación" y no sólo en "enseñanza"), pero inoculando "significativamente" las TIC como medio de incorporación del Ciberespacio a la escuela. Precisamente, en esta inoculación significativa de las TIC, el concurso de la Documentación se hace muy útil: el diseño instructivo de los elementos formativos de las TIC en el aula extendida, que acabamos de reseñar, debe servirse de los principios e instrumentos que la metodología de la Documentación en la gestión y análisis de contenido desarrolla en el marco de unas bibliotecas digitales educativas, convertidas en centros de recursos para el aprendizaje y la investigación.

\section{EL MODELO EDUCATIVO COMPETENCIAL Y LA ALFABETIZACIÓN EN INFORMACIÓN}

Las TIC y su eficaz inoculación en el sistema educativo mediante el aula extendida, por tanto, soportan adecuadamente un modelo educativo competencial, aun cuando la compleja realidad de la economía del conocimiento precisa de dos estilos muy complementarios en la vida formativa de cada ciudadano: un modelo de primacía educativa, con preeminencia de un magisterio que no sólo transmita conocimientos, habilidades y destrezas, sino "valores científicos" e investigación de base; un 
modelo de primacía enseñanza-aprendizaje, con preeminencia en las destrezas y habilidades técnicas en procedimientos e investigación aplicada. No cabe duda, que los dos "estilos formativos" se hacen presentes en la escuela del siglo XXI y se hacen patentes en la definición que, para las distintas titulaciones, el EEES hace del modelo competencial, clasificado en las competencias específicas de conocimiento, las competencias específicas profesionales, las competencias específicas académicas, las competencias transversales instrumentales, las competencias transversales personales y las competencias transversales sistémicas. En 1996, el informe de la OCDE “The Knowledge-based Economy”, apuntaba el desarrollo de la economía del conocimiento desde el aprendizaje continuo y las competencias informativas. En 1997, el programa PISA (Programm for International Student Assessment), acuñaba un concepto de alfabetización basado en la evaluación de la competencia lectora ${ }^{7}$, matemática, científica y de resolución de problemas. El proyecto DeSeCo (Definition and Selection of Competencies: Theoretical and Conceptual Foundations), en el mismo año, reconocía la alfabetización en información como una de las "competencias clave" de la competitividad económica y el desarrollo humano ${ }^{8}$.

Sin embargo, el modelo competencial de primacía educativa infiere una insoslayable "presencialidad escolar", por cuanto actúa sobre dos planos: uno técnico-procedimental, donde se adquieren y desarrollan las capacidades de dominio de herramientas digitales, aptitudes en los procedimientos virtuales y destrezas en la interactividad del aprendizaje colaborativo; otro cognitivo-inferencial, donde se garantiza la generación de conocimientos y saberes por el desarrollo de las capacidades en la selección y evaluación de los contenidos. Es precisamente en este plano, donde cobra protagonismo educativo la alfabetización en información y los instrumentos que le son propios, un plano donde la Documentación y, especialmente, el Análisis de Contenido prestan un servicio importante en la organización "alfabetizada" de la información para saber y conocer, en tanto que la Biblioteconomía, en la vertiente formativa de los bibliotecarios para los nuevos entornos tecnológicos, se convierte en un auxilio necesario en el diseño de las bibliotecas digitales educativas y la acción gestora de sus profesionales.

La formación en habilidades informativas orientadas a la formación ha sido, desde el siglo XIX una de las tareas encomendadas a la profesión bibliotecaria, de modo que las bibliotecas públicas, antes aún que las escolares, adquirían una importante función sustentadora en la alfabetización. Las distintas corrientes de renovación pedagógica, reflejadas en diferentes reformas educativas, integraban la biblioteca, en tanto que unidad de información para conocer, y las destrezas en la correcta utilización de sus partes constitutivas (catalogación, clasificación, 
búsqueda y recuperación, señalización, usos y divulgación informativa, extensión bibliotecaria), como elemento formativo básico. Las bibliotecas consideraron como una actividad prioritaria en sus funciones la formación de usuarios, incluso en algunas reformas curriculares en la escuela se comenzó a contemplar una materia de técnicas documentales. El crecimiento exponencial de la información en los años 70 del siglo XX propició en los países más industrializados ${ }^{9}$ una reflexión sobre la validez de las habilidades informativas tradicionales en el nuevo entorno socioeconómico, de modo que en paralelo a la formación de usuarios se abrió camino el concepto de alfabetización en información. En 1998 se fundaba en Estados Unidos el Institute for Information Literacy ${ }^{10}$, entre cuyos objetivos prioritarios se encuentran convertir los bibliotecarios en formadores de alfabetización en información y promover su integración curricular educativa. Por entonces SCONUL (Society of College, National and University Libraries desde 2001) aprobaba las "Aptitudes para el acceso y uso de la información en la enseñanza superior: la postura de Sconul"11, donde las habilidades de información se entendían como una convergencia entre la formación de usuarios bibliotecaria y la formación en nuevas tecnologías.

De las diferentes tentativas de normalización para la definición y puesta en práctica de estas habilidades durante la última década resultaron dos ensayos normativos realizados por distintas secciones de la ALA, las Information Literacy Standards for Student Learning (ALA y AETC, 1998) y las Information Literacy Standards for Higher Education (ACRL, 2000), que aceptados por instituciones educativas y organismos de certificación profesional, han extendido la pretensión de incorporar la alfabetización en información como un objetivo educativo primordial, especialmente en el espacio de la Educación Superior, por cuanto desde el congreso nacional estadounidense celebrado en 1987 "Libraries and the Search for Academic Excellence" la alfabetización en información se unía al concepto de aprendizaje permanente $^{12}$. Los años 90 del siglo XX además, con la eclosión de Internet, aportaron como decisiva novedad un nuevo concepto de búsqueda de información, otorgando prioridad al pensamiento del usuario a la tarea que debían realizar (que denomina "esfera cognitiva"), a los sentimientos unidos a la evolución de sus pensamientos ("esfera afectiva"), y a las acciones que realizaban y las estrategias que empleaban (“esfera física”).

Este breve repaso diacrónico de la evolución de la alfabetización en información demuestra cómo su definición conceptual ha comenzado "desde la base", a golpe de necesidades y respuestas profesionales, con un fuerte componente procedimental, de acción metodológica en planes institucionales, por lo que el principal esfuerzo de las iniciativas que deseaban transformar la alfabetización en información en un 
modelo integrado de planificación profesional y educativa se enfocó, naturalmente, hacia el logro de un consenso normativo. Así se entienden las "Information Literacy Standards for Higher Education" de la Association of College and Research Libraries (ACRL) de la ALA en el 2000, las "Normas sobre alfabetización en información" del Council of Australian University Librarians en 2001, o las Normas de ANZIIL (Australian and New Zealand Institute for Information Literacy) en 2004. Un esfuerzo similar se produjo a escala internacional para ensayar la convergencia de iniciativas en torno a la alfabetización en información, como muestran las pautas apuntadas por instituciones supranacionales como la UNESCO, la Unión Europea, pero muy particularmente la federación profesional bibliotecaria IFLA (Internacional Federation of Librarians Federations) desde 2002, una organización que contempla en una de sus secciones basada en la alfabetización en información. Ese año la "open session" se dedicó a la Developping librarians as teachers, reforzada por una "user education workshop" referida a la "information literacy"; en 2003. Pasadas proponía un certificado internacional para alfabetización en información, mientras la presidencia de la IFLA recogía entre sus temas clave "la alfabetización de por vida"; en 2004 la IFLA relacionó la alfabetización en información con la democracia. Este esfuerzo convergente internacional se vería reflejado en la aparición de la alfabetización en información, como oponente a la brecha digital, en las Cumbres de Ginebra y Túnez de la Sociedad de la Información y la publicación de los Faros de Alejandría en noviembre de 2005 con la alfabetización en información como actor indiscutible para el desarrollo hacia la Sociedad del Conocimiento.

El resultado de todo este reciente y activísimo proyecto ha sido que, sin una formulación epistemológica inicial sólida, el concepto de alfabetización en información sea muy transversal, poliédrico y multifacético según los escenarios. Budd, en 1997, había abordado una "alfabetización informativa", cuyos componentes eran la alfabetización visual, la alfabetización tecnológica, la alfabetización organizativa, la alfabetización mediática y la alfabetización cultural. En 2002 Bawden demostró la polisemia conceptual a partir de la propia terminología, por cuanto documentaba cómo la "alfabetización informacional" emerge unida a "alfabetización informática", "alfabetización electrónica", "alfabetización bibliotecaria", "alfabetización en medios", "alfabetización digital" ${ }^{3}$. Esta primera reflexión terminológica implicaba un acompañamiento en el fundamento conceptual, por lo que en paralelo se ha producido un esfuerzo investigador proyectado en tesis doctorales, comunidades virtuales (caso de MISTICA), blogs especializados como el diseñado por J.A. Gómez Hernández, grupos de investigación (Big Blue de las universidades de Manchester y Leeds; Universidad de Sheffield, dirigido por S. Webber; ENLIL, esto es, European Network on Information Literacy, UNAM en México; DOTEINE ${ }^{14}$ en la Universidad 
Carlos III de Madrid, dirigido por M.A. Marzal), grupos de trabajo (ALFINRED recientemente, 2007, constituido en España), congresos (las cinco ediciones, la última en 2006, del DHI en Ciudad Juárez; los e-Lit en Glasgow; los LOEX; LIASA en Suráfrica, entre otros), encuentros científicos y profesionales, como en el caso español dentro de los eventos FESABID, Congresos de Bibliotecas Públicas, ANABAD o REBIUN. Este complejo escenario conceptual justificó que, en febrero de 2006, aquellos investigadores y profesionales dedicados al desarrollo de un "modelo" de alfabetización en información se reuniesen y editasen la Declaración de Toledo ${ }^{15}$, un esfuerzo de normalización conceptual de un tópico de investigación, cada vez más importante.

En realidad la alfabetización en información es tan semánticamente polivalente como el propio concepto de "información", al que está referida, de forma que ha de ser entendida como una multialfabetización o conjunto de competencias transversales que actúan de forma cooperativa conforme el problema a resolver, por lo que en 2005 el National Forum on Information Literacy la ha definido como una constelación dinámica de competencias. No se trata de una competencia invasiva $\mathrm{e}$ inclusiva respecto de otras alfabetizaciones, competencias, habilidades y/o destrezas informativas, no se trata de un "conjunto" integrador de otros "subconjuntos" alfabetizadores, sino de un universo competencial bien integrado y estructurado que transforma al educando en un ser diestro y hábil en la gestión de contenidos para el conocimiento, en evaluación de contenidos para el saber, así como en la lectura digital y la escritura hipermedia. Aún más, este universo competencial se destina a cada usuario-educando de modo absolutamente individual: el individuo es el centro del proceso de aprendizaje y sujeto activo de su educación, que le proporcionará las herramientas intelectuales necesarias para construir su conocimiento a lo largo de la vida. En 2002, Bruce reconocía que la alfabetización en información descansaba sobre cuatro modos de aprendizaje, a saber, aprender a conocer o metaaprendizaje, aprender a hacer (competencia profesional y capacidad de adaptación), aprender a vivir juntos (empatía y sociabilidad) y aprender a ser (desarrollo pleno del individuo), todos los cuales deben ser la guía para la adaptación de los sistemas educativos al aprendizaje a lo largo de la vida.

\section{SELECCIÓN Y EVALUACIÓN DE CONTENIDOS: COMPETENCIA ALFIN EN EL MODELO EDUCATIVO}

La inoculación de la alfabetización en información como parte modular e integral de un modelo educativo competencial, tiene como razón justificativa última, sin duda, la eficacia del proceso de la información hacia el conocimiento. 
El planteamiento de esta hipótesis tiene una vertiente epistemológica, por la relevancia que los preconceptos y las operaciones intelectivas inferenciales tienen en la generación de conceptos y su asimilación en conocimiento, pero no cabe duda que tiene una vertiente funcional informativa, que es la que debe ocuparnos. En efecto, en Ciencias de la Documentación existe un acuerdo tácito de que el documento, con independencia de su soporte, mensaje, código o usuario, es una función, esto es, la información cifrada en el documento adquiere una dimensión para el conocimiento diferente según para qué vaya a servir y el contexto de su aplicación. La información documental, pues, no tiene una materia propiamente dicha, sino que tiene un interés para el lector, por tanto los contenidos informativos poseen una potencialidad epistemológica para el conocimiento, acusada por la transversalidad, dinamicidad, politextualidad, granularidad y multidisciplinariedad de los datos en un hipermedia. Estos caracteres, por lo demás, se adecuan perfectamente al funcionamiento de las comunidades virtuales, donde prima un aprendizaje por tópicos (no materias) y mediante un aprendizaje cooperativo.

El carácter poliédrico de los datos hipermedia hace que sea sumamente importante que el sistema de información en Educación y los propios educandos tengan competencias para seleccionar y evaluar los contenidos digitales informativos más adecuados a sus fines de conocimiento. Las bibliotecas, en su proceso de transformación a "bibliotecas híbridas" por conversión en bibliotecas digitales educativas, en donde debe radicarse la acción de la alfabetización en información, deben atender a dos principales variables:

- La preeminencia del usuario, de modo que una biblioteca digital es sobre todo personalizada. El usuario, mediante su tópico e interés, dispone de forma personal la colección digital conforme a sus necesidades concretas de conocimiento. El usuario, pues, debe desarrollar unas competencias de alfabetización para seleccionar y evaluar los contenidos digitales pertinentes a su interés cognitivo, si bien la biblioteca digital debe poseer los instrumentos adecuados para satisfacer estrategias de conocimiento individuales y adaptadas a un tipo de "pensamiento asociativo".

- La preeminencia de la selección y evaluación de los contenidos digitales por parte de la biblioteca, como sistema de información para Educación. La Documentación ha tenido definidos principios y criterios de selección de los documentos por sus contenidos, sin embargo en los nuevos entornos tecnológicos se hace preciso el diseño de un modelo evaluativo (previa selección inicial) capaz de organizar los datos por criterios de eficacia en el proceso 
educativo, pero además capaz de integrar un conjunto de variables estadísticas en tanto que indicadores, para determinar el grado de eficacia de la aplicación del modelo en la comunidad educativa.

El desarrollo de habilidades y destrezas en la selección y evaluación de contenidos conforma un objetivo competencial importante para la alfabetización en información, pero siempre atendiendo a dos dimensiones derivadas de la acción del educando o del recurso, dentro del modelo competencial educativo en una biblioteca. Deben destacarse dos sistemas evaluativos, cada uno diferente según la competencia de alfabetización en información que se reclame al educando:

\section{Modelo competencial evaluativo sobre los contenidos}

Pretende evaluar la eficacia de los contenidos de los recursos digitales de información para la generación de conocimiento sobre el educando. Naturalmente, el modelo tiene que ver con la accesibilidad y usabilidad del recurso. La accesibilidad, a partir de lo que en Europa se conoce como Design for all, tiene que ver con la facilidad técnica e intelectual de uso y comprensión del recurso, que pueden verse perturbadas por: el diseño del software, por una inadecuada visualización desde los sistemas operativos a los navegadores; por el hardware, por el diseño técnico de la propia herramienta; por el diseño de la información, según esté dispuesta. La usabilidad, de otro lado, está determinada por la verificación de la amigabilidad, flexibilidad, diseño multimedia, virtualidad e interactividad de los contenidos ${ }^{16}$. La accesibilidad debe tener una relación directa con la usabilidad del recurso ${ }^{17}$.

Esta breve reflexión inicial, sobradamente conocida, es muy útil, sin embargo para que la adecuada relación de la accesibilidad-usabilidad, en una correcta arquitectura de la información, garantice que el contenido de un recurso de información sea eficaz para la generación de conocimiento en el educando. Los criterios evaluativos deben basarse, entonces, en los mecanismos de la cognición humana, que para los sistemas de información son particularmente relevantes:

La percepción, operación por la que un individuo hace una selección de los estímulos registrados, a partir de estímulos como la novedad, la incertidumbre, el conflicto y la complejidad. La percepción se relaciona, pues, con la usabilidad de los contenidos en la Web, bien por la legibilidad lingüística referida en el recurso, bien por la legibilidad visual respecto de la descodificación del mensaje del recurso. La percepción, en todo caso, está determinada por la accesibilidad, requisito que todo documento debe satisfacer para todo usuario. 
La atención, que filtra y organiza la información para que el individuo formule respuestas adecuadas en una acción concreta. Se distinguen diferentes tipos de atención, para nuestro objetivo nos interesa destacar la atención distribuida y espacial, en la que el individuo atiende un campo perceptivo la espera de estimulo que le atraiga para dirigirse a él. La atención del lector es más efectiva por la coherencia estilística de los sitios, o, en caso de páginas cuyos tiempos de carga presenten alguna demora, anticipar la visualización de su estructura. Esto determina la importancia de la focalización de la atención, pues la fijación del foco es condición para una atención selectiva, que consiste en la habilidad de concentrar la atención en un canal que contiene informaciones presentadas con elementos de distracción. La atención selectiva evita que el sistema cognitivo se sobrecargue de información.

La memoria destinada a adquirir, conservar, recuperar y utilizar conocimientos y habilidades. El modelo clásico de Atkinson y Shiffrin describe la memoria como un sistema organizado en tres etapas: sensitiva, a corto plazo y a largo plazo. Tras la experiencia sensitiva, se pasa a la etapa de memoria a corto plazo, que evita la sobrecarga cognitiva, y la memoria a largo plazo detenida en la información que no está siendo elaborada. Este tipo de memoria ofrece un acceso a la información en ocasiones difícil, requiriendo mecanismos que faciliten la recuperación. La memoria a largo plazo se distingue la memoria declarativa, que almacena la información según criterios fácticos o conceptuales y según formatos que pueden ser verbales o simbólicos; y la memoria procedimental, que almacena los procedimientos siguiendo las reglas de producción.

A partir de estos mecanismos cognitivos Han et al ${ }^{18}$ diseñaron una puesta de criterios de evaluación de la usabilidad, con 48 posibles dimensiones de la usabilidad, clasificadas en dos grupos: las relacionadas con el uso del producto (categorizados según el estadio cognitivo de procesamiento de la información implicado) y las relacionadas con la percepción del usuario respecto al producto, una propuesta refinada por Busetti que reconoce 23 criterios de evaluación agrupados en torno a tres categorías: captación, vinculada al mecanismo cognitivo de la atención; fidelización, vinculada a la percepción; capacidad alfabetizadora, vinculada con la memoria.

\section{Modelo competencial evaluativo sobre el usuario}

Pretende evaluar el impacto sobre la eficacia cognitiva de la acción del usuario sobre los contenidos de los recursos, a partir de una correcta disposición infraestructural y organizativa de la institución educativa. En este caso, el modelo evaluativo se orienta a medir el progreso de los logros y acciones destinadas a la 
consecución de los objetivos educativos propuestos. El modelo evaluativo debe facilitar la toma de decisiones para un modelo competencial de Educación de calidad, pero además inducir a los agentes educativos a una actuación en la consecución de los objetivos propuestos en un nivel de planificación estratégica. El modelo, pues, debe permitir evaluar comparativamente, mediante el ejercicio del benchmarking los progresos hacia la Sociedad del Conocimiento, para así fundamentar un sistema educativo de calidad. El compromiso por la calidad se ha convertido en uno de los elementos básicos para un modelo educativo competencial, un compromiso que se ha plasmado en el desarrollo de indicadores. Estos indicadores se han diseñado, en principio, para la medición estadística cuantitativa de la infraestructura educativa, si bien el mismo modelo educativo competencial ha impulsado un desarrollo conjunto de indicadores que permitan una información más cualitativa sobre los progresos derivados de proyectos y programas educativos.

Un caso prototípico se plantea en la Unión Europea, muy activa en el diseño y aplicación de un modelo educativo competencial a través del Espacio Europeo de Educación Superior, mediante el Statistical Indicators Benchmarking Information Society (en adelante SIBIS), como marco de desarrollo de indicadores. El proyecto se inició en 2001 y finalizó en 2003, siendo sus objetivos primordiales generar un marco conceptual y metodológico para aplicar benchmarking, revisar y verificar conceptos y fuentes de información estadísticos, con el fin de desarrollar nuevos indicadores para medir el impacto de las acciones encaminadas al desarrollo de la Sociedad del Conocimiento, especialmente en el ámbito de Telecomunicaciones, acceso, seguridad, competencias laborales, inclusión social, comercio, salud y administración electrónicas, educación e investigación. SIBIS buscó evaluar la calidad y pertinencia de los nuevos indicadores que proponía, para lo que tuvo en cuenta: cada indicador debía contar con una argumentación teórica que relacionase con coherencia el indicador con el concepto a medir; capacidad del indicador para medir lo que se pretende que mida; la fiabilidad, referida a la necesidad de que el indicador produzca iguales resultados, independientemente de donde se aplique; disponibilidad y accesibilidad de los datos tratados por los indicadores.

El modelo evaluativo puede sustentarse conceptualmente en el Cuadro de Mando Integral (Balanced Scorecard), instrumento de gestión y control basado en indicadores y herramienta que permite estructurar una estrategia en la organización (la institución educativa), como conjunto de líneas de actuación para la consecución de objetivos propuestos. El Cuadro de Mando Integral (en adelante CMI) recoge en un cuadro sintético el conjunto de indicadores, fomentando la eclosión de una conciencia estratégica en la institución para relacionar resultados a corto y largo plazo, 
vigilando procesos y resultados, que impulsan acciones futuras y permiten respuestas adecuadas, integrando de modo sistémico y preciso indicadores cuantitativos y cualitativos, entre otros, que puedan proyectarse en el rendimiento organizativo ${ }^{19}$. La aplicación del CMI permitiría trasladar a las instituciones educativas los objetivos educativos estratégicos de alto nivel, generando un sistema de indicadores de rendimiento en áreas educativas críticas, que combinase indicadores referidos a la eficiencia y productividad, aspectos corporativos, percepción externa de calidad y el valor de la acción.

\section{MÉTODOS DIDÁCTICOS COMPETENCIALES HACIA LA ALFABETIZACIÓN EN INFORMACIÓN}

Un eficaz impacto educativo de alfabetización en información sobre la formación competencial del educando debe tener una demostración de efectos. Esta demostración debe efectuarse sobre dos competencias específicas: la captación competente de contenidos educativos pertinentes, mediante el control hábil de instrumentos de gestión de contenidos para navegar comprensivamente entre la asociatividad de los recursos de información educativa; la edición eficaz de contenidos digitales educativos, para representar conocimientos adquiridos y que implican una destreza intelectiva. Es evidente que ambas competencias instructivas tienen como espacio idóneo de desarrollo en bibliotecas digitales educativas.

La captación comprensiva de recursos de información educativos, implica una competencia en lectura digital para recuperar y estructurar argumentativamente los recursos digitales: las posibilidades que, para esta competencia lectora, suponen los mapas conceptuales como asignación significativa de recursos web a un tópico, los tópic maps como visualización y diseminación significativa de recursos web, o los tesauros con una potente carga asociativay visual para capturar recursos politextuales, son elementos constitutivos muy relevantes en las bibliotecas digitales educativas. La edición, en tanto que escritura hipermedia por representación de contenidos, se canaliza en las bibliotecas digitales educativas mediante los metadatos educativos, que hacen compatibles e interoperables los objetos digitales con vocación educativa editados, la tecnología wiki, que soporta eficazmente la edición de documentos colaborativos y en permanente revisión, los blogs por su carácter idóneo para la secuenciación de recursos web utilizados en una programación didáctica y la edición de trabajos digitales por la sindicación de contenidos, así como también los objetos de aprendizaje, con sus caracteres educativos formales e intrínsecos. En un principio los ensayos hacia una metodología didáctica competencial han fijado su atención en la operación de aprendizaje del proceso educativo, esto es, la 
edición de un "recurso educativo", entendido como cualquier material que, en un contexto educativo determinado, sea utilizado con una finalidad didáctica o para facilitar el desarrollo de las actividades formativas ${ }^{20}$ y materiales didácticos web, entendiendo por tales aquellos diseñados digitalmente como materiales curriculares para el proceso de enseñanza-aprendizaje en entornos tecnológicos ${ }^{21}$. En el modelo educativo competencial son particularmente significativos los objetos de aprendizaje y los blogs.

Desde el punto de vista de la Documentación, apoyada en la "Arquitectura Instruccional", la edición de objetos de aprendizaje precisa cuatro instrumentos: descubrimiento, para dotar al educando de una línea para iniciar la búsqueda por uno o más objetos de aprendizaje; presentación, inspección, recomendación, triple funcionalidad que dispone los objetos de aprendizaje obtenidos en la búsqueda según el orden estimado oportuno para el educando, un algoritmo inspecciona los criterios de búsqueda y preferencia del educando, por fin el instrumento recomienda información al remitir al educando hacia otros objetos de aprendizaje con contenidos afines o complementarios al seleccionado; combinación, que secuencia los objetos de aprendizaje, con distintas posibilidades para contextualizar el aprendizaje; reflexión, para captar la interacción del educando con los objetos de aprendizaje y con otros educandos de similar perfil. La adscripción de los objetos de aprendizaje a una Arquitectura Instruccional ha permitido iniciar un proceso de precisión conceptual hasta llegar a definiciones tan acertadas como la de L'Allier ${ }^{22}$, que los entiende como la unidad estructural más pequeña e independiente que contiene un objetivo, una actividad o una evaluación para el aprendizaje. El autor entiende por objetivo un componente estructural que describe los criterios para la consecución de unos resultados previstos en una actividad. Actividad, referida siempre a un objetivo y entendida como el componente que inocula un aprendizaje. Evaluación, criterio e indicador que determina si un objetivo ha sido logrado. De este modo, los objetos de aprendizaje tienen como elementos básicos de definición:

- $\quad$ Propiedades predicativas, que incluyen: en primer lugar la intención de aprendizaje, definida por dos "aspectos característicos", la forma, conjunto de rasgos y caracteres que permiten percibir, asimilar y comprender el objeto de aprendizaje, y la relación, proceso inferencial para transformar el objeto de aprendizaje en conocimiento, un aspecto vertebrado por el discurso, capaz de dotar de una lógica razonada la información contenida en el objeto de aprendizaje; en segundo lugar, la reusabilidad, que supone establecer una separación entre creación y difusión de un objeto de aprendizaje, de modo que pueda estar dispuesto para ser aplicado en distintas situaciones instructivas. 
- Funcionalidad, definida porla accesibilidad, a partir de normas, especificaciones e implementaciones que soporten la compatibilidad e interoperabilidad de los objetos de aprendizaje (tal es el caso de Learning Object Metadata Working Group, el IMS Global Learning Consortium, el Dublín Core Metadata Iniciative, los LOM de IEEE, SCORM); la interoperabilidad, sistema colaborativo que filtra la información durante la colecta y la propagación de los componentes instructivos de los objetos de aprendizaje, generando una ordenación de prioridades según la evaluación dada por los educandos; aplicabilidad, servicios que puede prestar un objeto de aprendizaje en un entorno educativo, mediante la incorporación de tutores, expertos, grupos de aprendizaje colaborativo, situaciones de aprendizaje, actividades de aprendizaje, materiales de aprendizaje, medios tecnológicos de ejecución, estructuras conceptuales, ideas instructivas, feedback.

Los blogs, de otro lado, tienen una potencialidad educativa importante. La tecnología que subyace detrás de los blogs es RSS, un sublenguaje surgido de la aplicación de XML, diseñado para la distribución (syndication en inglés) de noticias o información tipo noticias contenidas en sitios web. Los archivos RSS comúnmente se llaman feeds RSS o canales RSS y contienen un resumen de lo publicado en el blog de origen, estructurándose en uno o más ítems. Cada ítem consta de un título, un resumen de texto y un enlace a la fuente original en el blog donde se encuentra el texto completo. Además pueden incluir información adicional como el nombre del autor o la fecha y la hora de publicación del contenido. El agregador permite ver los contenidos de múltiples blogs sin necesidad de visitarlos individualmente. Todas estas posibilidades técnicas demostraron su eficacia en entornos educativos: la publicación de contenidos se asemeja por su facilidad a los correos electrónicos; su sistema de comentarios facultan para una interactividad con los visitantes del blog, quienes además pueden suscribirse al blog y recibir las novedades mediante los agregadores de RSS, que están en línea o integrados en algunos navegadores aún más mediante un agregador el usuario puede suscribirse a un canal de RSS, como sindicación, de modo que cada espacio de tiempo marcado el agregador revisa los contenidos en web, proporcionando constantemente una actualización sobre un tópico de interés a través del canal ${ }^{23}$, generando así un repertorio de enlaces permanentes a los posts (los textos en el blog) con una vinculación desde otros sitios web que garantiza la secuencia de navegación.

Los blogs son un instrumento idóneo para una alfabetización tecnológica, por cuanto no precisan unos sofisticados conocimientos informáticos pero son muy asequibles para escribir al editar texto, y leer comprensivamente textos y documentos 
relacionados por enlaces y sindicaciones, así como por manifestarse como un espacio muy adecuado para la "narración de historias", una destreza de competencia lecto-escritora de ficción sumamente importante en el proceso alfabetizador ${ }^{24}$. Los blogs, además, poseen un notable potencial instructivo: por la precisión, pues los posts exigen una escritura concisa para asegurar una lectura rápida comprensiva; la estructuración del discurso, pues permite una ordenación diacrónica de los textos; la interactividad, al actuar como una sencilla plataforma digital para una efímera comunidad virtual entre condiscípulos; su semiótica multimedia, ejercita la lectura y escritura icónicas y virtuales; la inmediatez en la comunicación permite una interactividad asíncrona y la adaptación curricular, por superar las limitaciones espacio-temporales del aula, lo que permite sustentar un ritmo de aprendizaje individualizado ${ }^{25}$ y de desarrollar el aula extendida.

\section{CRAI Y LA DOCENCIA COMPETENCIAL HACIA LA ALFABETIZACIÓN EN INFORMACIÓN}

De un modo fragmentado, a veces intuitivo y otras por las exigencias dispares de todos los agentes críticos involucrados en la Educación, las necesidades de radical transformación del modelo educativo iban hallando distintos instrumentos de respuesta, algunos de los cuales hemos ido detallando. En la Unión Europea se fue materializadon en el Espacio Europeo de Educación Superior, que iniciaba su andadura como modelo en 1998, pero ha ido cobrando cuerpo real y aplicado en las universidades europeas en la actualidad ${ }^{26}$. Este Espacio, entre otras cosas, hacía una aportación fundamental: el sistema, que incorpora de forma significativa e integrada las TIC pero apostando por la presencialidad (la enseñanza descansa en las TIC, la Educación en la socialización del espacio universitario), para su desarrollo necesita otro tipo de biblioteca universitaria, reclamando una transformación. Esta transformación no se detenía sólo en la apuesta de una biblioteca digital educativa, sino pretendía un Centro de Recursos para el Aprendizaje y la Investigación.

Es importante resaltar la noción básica de "centro de recursos", por cuanto supone centralizar en un servicio sistémico y estructurado todos aquellos recursos (documentales, infraestructurales, ciberespaciales) pertinentes en una Educación para la Sociedad del Conocimiento. En este contexto, la biblioteca universitaria debía hacer un doble esfuerzo consecutivo: la confluencia con los servicios informáticos y audiovisuales de la universidad y, luego, la convergencia académica y administrativa en el organigrama universitario, para centralizar lo recursos para sostener los nuevos aprendizajes e investigaciones. Este esfuerzo infería dotar a las 
bibliotecas universitarias en su transformación a CRAI de un potente equipamiento para captar recursos y digitalizar en archivo abierto, pero sobre editar (por parte de profesores, investigadores y alumnos) el material adecuado para los nuevos entornos tecnológicos y en formatos técnicos sofisticados para su diseminación y publicación ${ }^{27}$. El gran salto cualitativo convergente está actualmente en sus inicios, como se recoge en el nuevo Plan Estratégico de REBIUN 2007-2010: la función del CRAI en alfabetización en información. Han comenzado a ponerse en práctica, en España, diferentes métodos de desarrollar competencias de alfabetización en información desde bibliotecas universitarias en transformación a CRAI para sus comunidades educativas, sin embargo este notorio esfuerzo hace más patente la necesidad urgente de asentar un modelo conceptual claro de alfabetización en información en el currículo universitario (alternativo a la formación de usuarios), para plasmarse en una programación y metodología para cursos competenciales en alfabetización en información, todo ello condicionantes preliminares básicos para diseñar cursos propios en plataformas digitales educativas, como moodle, editar objetos de aprendizaje, comunidades virtuales con sus instrumentos y documentos imprescindibles, sistemas de evaluación competencial y modos de acreditación en estas competencias, dentro de una red cooperativa.

Siguiendo todos los condicionantes detallados a lo largo del artículo, existe una experiencia programática en la materia "Análisis de Contenido", del $2^{\circ}$ curso de la Licenciatura en Documentación de la Universidad Carlos III de Madrid, conforme a criterio del Espacio Europeo de Educación Superior, que ha orientado su programación a los servicios que podría procurar el CRAI. La Programación detalla:

- Competencias. Entendidas como un adiestramiento intelectivo, que garantice el dominio experto de los principios teóricos de la materia para el correcto ejercicio del diseño de sistemas de información, la facultad para el aprendizaje continuo y la readaptación a las sucesivas innovaciones profesionales:

- Conocimiento de la naturaleza de la información en los nuevos entornos tecnológicos, así como de su ciclo de producción y transferencia hacia la Web semántica.

- Conocimiento de los principios teóricos para la organización, representación y recuperación de contenidos en los recursos informativos.

- Conocimiento de las tendencias de investigación, en modelos e instrumentos, para el procesamiento de contenidos hacia la web del conocimiento. 
- Conocimiento de los principios para el análisis en la producción, transferencia y uso de los recursos informativos para la actividad científica $\mathrm{y}$ aprendizaje en las organizaciones.

- Conocimiento de las tecnologías de la información para el eficaz diseño de sistemas de información en la gestión de contenidos para la gestión del conocimiento.

- Conocimiento de estrategias para el desarrollo del razonamiento crítico en la valoración y análisis de alternativas, así como en la propuesta de mejoras e innovaciones.

- Desarrollo de estrategias para el aprendizaje autónomo en la adaptación a los cambios del entorno, así como de integración en equipos multidisciplinares de trabajo.

- Habilidades. Entendidas como un adiestramiento técnico, que garantice el dominio experto de los principios metodológicos de la materia para el correcto ejercicio profesional de las tareas en las unidades de información:

- Capacidad para aplicar las técnicas, normativas e instrumentos propios para la organización, representación y recuperación de los contenidos en los recursos informativos.

- Capacidad y habilidad en el manejo de tecnologías de la información en el proceso de producción, transferencia y uso de contenidos en los recursos informativos.

- Habilidad para analizar las necesidades informativas, asesorar en los modos y medios de acceso al contenido y formar-alfabetizar en información a los usuarios.

- Habilidad en la obtención, tratamiento e interpretación de contenidos informativos para su evaluación y selección en los procesos de transferencia y uso.

- Capacidad y habilidad en la aplicación de instrumentos para el desarrollo de contenidos en los sistemas de información.

- Habilidades en el uso de software genérico.

- $\quad$ Capacidad para organizar y planificar el propio trabajo. 


\section{- Objetivos didácticos generales}

- Comprender las transformaciones en la naturaleza de documento y contenido en los nuevos entornos tecnológicos y que alteran la finalidad y función del Análisis Documental de Contenido.

- Conocer y comprender las tendencias de modelos teóricos y aplicativos de Análisis de Contenido que se derivan en lenguajes documentales.

- Capacitar en la manipulación experta de los lenguajes documentales para la organización, representación y recuperación del contenido en los recursos informativos.

- Capacitar en la manipulación experta de la aplicación de las tecnologías de la información para el desarrollo de los nuevos instrumentos de análisis de contenido y lenguajes documentales.

- Conocer las estrategias para la representación del contenido y su traducción y derivación en documentos secundarios, así como las estrategias de la organización del contenido para su uso en el conocimiento de lenguajes de especialidad.

- Capacitar y habilitar para el análisis de las necesidades informativas del usuario determinando la evaluación dela representación del contenido para la satisfacción de esas necesidades dentro del sistema de información.

- Capacitar y habilitar en la aplicación de los lenguajes documentales dentro de las unidades de información para el desarrollo de la representación y recuperación de contenidos.

- Capacitar para organizar y planificar el trabajo de análisis de contenido en una unidad de información.

El desarrollo didáctico ha aprovechado uno de los servicios de la plataforma digital Aula Global. Cada materia en su menú tiene cuatro enlaces importantes, con sus posibilidades, permitiendo interpretar aquellos enlaces que puedan ser más afines a los objetivos competenciales:

- Información, donde incluye: la Ficha de la Asignatura, en la que se aloja la programación didáctica; la Bibliografía, que enlaza directamente al OPAC de la biblioteca de la universidad, mostrando al alumno dónde se halla la bibliografía recomendada y permitiéndole realizar las acciones que el OPAC le permite (reserva, préstamo, renovación, etc.). 
- Materiales de estudio, donde son muy útiles: unidades didácticas, corpus en pdf de la "doctrina", cerrada y estructurada de la materia, que sustituyen los apuntes y permite volcar los contenidos una semana antes de iniciar la unidad para estar leídos por los alumnos cuando se inicie su consideración en la clase presencial; materiales complementarios, elenco de URLs de documentos científicos que ilustran, contraponen, explican, desarrollan, contradicen los apartados de la unidad didáctica, también colgados al tiempo que ellas, para que el alumno tenga una visión alternativa a la "ciencia cerrada" de la unidad didáctica; recursos de información, donde, mediante un mapa conceptual, se visualizan todos los tópicos de la materia y se le adjuntan URLs de revistas, asociaciones profesionales, grupos de investigación, muy destacados en el tópico, modo de asegurar al alumno el aprendizaje permanente.

- Actividades, con tres partes bien delimitadas e importantes: ejercicios, donde se agregan instrumentos gratuitos en red y software libre para que, en clase, los alumnos practiquen los conceptos vistos en la unidad didáctica y los materiales complementarios; prácticas, son propuestas de experiencias aplicativas de cada unidad didáctica, una propuesta que el alumno debe hacer en el laboratorio informático y que están diseñadas siguiendo los caracteres de los objetos de aprendizaje, estas dos partes acreditan la dimensión competencial de la materia; debates, bajo un tópico concreto, que se transforma en un foro, pero con la intención de ser utilizado como comunidad virtual para el aprendizaje cooperativo.

- Tutoría y comunicación, espacio reservado para el seguimiento individual del alumno, un criterio básico en este modelo educativo competencial.

\section{CONCLUSIONES}

La argumentación plasmada a lo largo de esta exposición nos lleva a destacar, como elementos para "desarrollos futuros" de investigación, unas conclusiones a modo de reflexión:

- $\quad$ Es preciso incorporar el innegable potencial educativo del Ciberespacio a la institución escolar, como medio para hacer recuperar a la escuela su función nodal en Educación, esfuerzo en el que la Documentación puede ser muy útil, por proporcionar instrumentos de gestión de contenidos que optimicen la eficacia de las aulas extendidas en una "alfabetización ciberespacial". 
- La nueva "alfabetización ciberespacial" debe entenderse como una pericia selectiva en la lectura y escritura digitales, para mejor inferir conocimiento desde la información, por lo que se denomina alfabetización en información, siendo su medio más significativo las competencias, conforme al concepto que se ha ido definiendo y es implícito en el EEES.

- Las competencias tienen su espacio educativo idóneo en las aulas extendidas, por acoger con la mayor relevancia didáctica los más atractivos instrumentos para el aprendizaje colaborativo (blogs, wiki, objetos de aprendizaje, mapas conceptuales, tópic maps, ontologías).

- El verdadero valor educativo de las TICs se proyecta en un modelo de "educación presencial", donde las TICs no son un medio de formación virtual, sino un instrumento para ejercitar el aprendizaje permanente, complementado por una "socialización educativa", que debe estar garantizada por espacios para "personalizar" el aprendizaje colaborativo, los CRAI. El valor educativo de las TICs sólo será posible mediante su uso experto, función que corresponde a la alfabetización en información.

- La alfabetización en información, pues, debe entenderse como una competencia genérica, que procura una habilidad excelente para la evaluación de los contenidos digitales educativos en un espacio educativo muy específico, las bibliotecas digitales educativas, que desembocan en su conversión en CRAI.

- La absoluta relevancia de la evaluación en un modelo educativo competencial, mediante la alfabetización en información, debe practicarse sobre dos planos: la usabilidad en contenidos, a partir del desarrollo de competencias en percepción, atención y memoria; la capacidad del usuario, a partir de un seguimiento analítico mediante "indicadores cualitativos", que comenzó a apuntar el modelo SIBIS.

- La alfabetización en información, por su naturaleza competencial en entornos tecnológicos, tiene su propia metodología didáctica para convertirse en un verdadero instrumento de Educación para la Sociedad del Conocimiento: el método que determina el uso de los objetos de aprendizaje y los blogs, con unos caracteres y potencialidades muy precisos.

- Los CRAI, convertidos en el espacio educativo "bibliotecario" para el nuevo modelo educativo competencial, deben acreditar su verdadera dimensión 
formadora en alfabetización en información, superando su mera funcionalidad como biblioteca digital educativa, por lo que no sirve su simple transformación en portales de información educativa o gestores de recursos web, sino que deben integrarse intrínsecamente en el nuevo proceso educativo, a través de experiencias, como la que se ha apuntado.

\section{NOTAS}

1 Centro de Recursos para el Aprendizaje y la Investigación.

2 Bunk, G. P. (1994). La transmisión de competencias en la formación y perfeccionamiento profesionales en la RFA. Revista Europea de Formación Profesional, (1), 8-14.

3 Le Boterf, G. (2000). Construire les competentes individuelles et collectives. París: Editions d'Organisation.

4 Colás Bravo, M. (2005). La formación universitaria en base a competencias, en: Colás Bravo, T.; De Pablos Pons, J. (coords.) La Universidad en la Unión Europea. Málaga: Imagraf.

5 Lévy-Levoyer, C. (2003). Gestión de las competencias. Barcelona: Gestión 2000 S. A.

Martínez, D.; Martí, R. (2003). La factoría de recursos docentes, en: Los Centros de Recursos del Aprendizaje y la Investigación en los procesos de innovación docente: Jornadas Rebiun 2003. [en línea] Disponible en: http://biblioteca.uam.es/paginas/ palma.html [consulta 2007, 2 de mayo]

Cuevas Cerveró, A.; Vives i Gràcia, J. (2005). La competencia lectora en el estudio PISA: Un análisis desde la alfabetización en información. Anales de documentación, (n $\left.{ }^{\circ} 8\right), 51-70$.

OCDE (2005). Definition and Selection of Competencies: Theoretical and Conceptual Foundations. Executive Summary [en línea] Disponible en: http://www.oecd.org/ dataoecd/47/61/35070367.pdf [consulta 2007, 10 de mayo].

Es el caso de Estados Unidos, donde la information literacy ha tenido su primera eclosión. Bernhard, P. (2002). La formación en el uso de la información: una ventaja en la enseñanza superior. Situación actual. Anales de documentación, no ${ }^{\circ}$, 409-435. [en línea] Disponible en: http://www.ala.org/acrl/nili/charge.htm [consulta 2007, 12 de mayo].

11 La traducción al castellano de este informe, originalmente fechado en 1999, fue publicada en 2001 por el Boletín de la Sociedad Andaluza de Bibliotecarios, Número 62, Vol (16), 63-77.

Breivik, P. S.; Gordon, G. E. (1989). Information Literacy: Revolution in the Library. Phoenix: American Council on Education and Oryx Press.

13 University of South Florida, Directory of Online Resources for Information Literacy: Definitions of Information Literacy and Related Terms [en línea]. Disponible en: http://bulldogs.tlu.edu/mdibble/doril/definitions.html [consulta 2007, 9 de mayo]. Ofrece definiciones de los términos information literacy, information competence, media literacy, computer literacy, visual literacy, lifelong learning y resource-based learning, entre otros. 
Marzal, M. A. Documentación y Tecnologías de la información para educación: Herramientas para la alfabetización informacional y organización de recursos didácticos (DOTEINE). Entidad financiadora: Ministerio de Ciencia y Tecnología. Referencia BSO2003-04895 [en línea] Disponible en: http://travesia.mcu.es/S ALFIN/ficheros/Declaracion Toledo.pdf. [consulta 2007, 12 de mayo] Hizo una definición parcial, Millar, R. H. (2002). E-learning site usability checklist. [en línea] Disponible en: http://www.stcsig.org/usability/resources/toolkit/elearning-checklist.doc2002 [consulta, 2007, 9 de mayo]

Una interesante relación se encuentra en Martínez Normand, L. (2001). Valoración de la accesibilidad y usabilidad de las páginas web españolas. VI Jornadas SIDAR 2001. [en línea] Disponible en: http://www.sidar.org/acti/jorna/6jorna/ponen6/ loic/ [consulta 2007, 9 de mayo]

Han, S. et al. (2001). Usability of consumer electronic products. International Journal of Industrial Ergonomics, vol. 28, ( n. 3-4), 143-151.

Jorge, C. (2002). Los indicadores de rendimiento como guía de la gestión: aportaciones del Balanced Scorecard a las bibliotecas. Forinfa, 17 (julio-septiembre). [en línea] Disponible en: http://lemi.uc3m.es/forinf@/index.php/Forinfa/article/view/76 [consulta 2007, 12 de mayo]

Definiciones tomadas de Marqués Graells, P. (2007). Los medios didácticos.[en línea] Disponible en: http://dewey.uab.es/pmarques/medios.htm [consulta 2007, 10 de mayo]

Define y caracteriza Area, M. (2003). De los Webs educativos al material didáctico Web. Comunicación y Pedagogía. ( $n^{0}$ 188), 32-38. L'Allier, J. J. Frame of Reference: NETg's Map to the Products Their Structure and Core Belief. [en línea] Disponible en: http://www.netg.com/research/whitepapers/ frameref.asp [consulta 2007, 10 de mayo] Argumenta Placita, F. (2005). Aplicaciones de los weblogs para la enseñanza en línea. [en línea] Disponible en: http://www.hets.org/vp es weblogs.php [consulta 2007, 11 de mayo] Huffaker, D. (2004). Spinning yarns around a digital fire: Storytelling and dialogue among youth on the Internet. First Monday, 9, (1), [en línea] Disponible en: http:// www.firstmonday.org/issues/issueg 1/huffaker/ [consulta 2007, 11 de mayo] Kajder, S.; Bull, G. (2003). Scaffolding for Struggling Students: Reading and Writing with Blogs. Learning\&Leading with Technology, 31, (2), 32-35.

Sus caracteres y evolución es muy prolija y excede este trabajo, pero se pueda consular un nodo muy ilustrativo [en línea] Disponible en: http://www.uc3m.es/uc3m/eees/ [consulta 2007, 11 de mayo] Un panorama suficiente del esfuerzo transformador se recoge en la web de las IV Jornadas REBIUN. (2006). IV Jornadas CRAI. Universidad de Burgos.[en línea] Disponible en: http://www.ubu.es/biblioteca/crai// [consulta 2007, 11 de mayo] 


\section{REFERENCIAS BIBLIOGRÁFICAS}

Area, M. (2003). De los webs educativos al material didáctico web. Comunicación y Pedagogía. $n^{0}$ 188, 32-38.

Bernhard, P. (2002). La formación en el uso de la información: Una ventaja en la enseñanza superior. Situación actual. Anales de documentación, $\mathrm{n}^{0} 5$, 409435 .

Breivik, P. S.; Gee, E. G. (1989). Information Literacy: Revolution in the Library. Phoenix: American Council on Education and Oryx Press.

Bunk, G. P. (1994). La transmisión de competencias en la formación $y$ perfeccionamiento profesionales en la RFA. Revista Europea de Formación Profesional, 1, 8-14.

Colás Bravo, M. (2005). La formación universitaria en base a competencias. En Colás Bravo, T.; de Pablos Pons, J. (coords.). La Universidad en la Unión Europea. Málaga: Imagraf.

Cuevas Cerveró, A.; Vives i Gràcia, J. (2005). La competencialectora en el estudio PISA: un análisis desde la alfabetización en información. Anales de documentación, $\mathrm{n}^{\circ} 8,51-70$.

Han, S. et al. (2001). Usability of consumer electronic products. International Journal of Industrial Ergonomics, vol. $28, n^{0} 3-4,143-151$.

Huffaker, D. (2004). Spinning yarns around a digital fire: Storytelling and dialogue among youth on the Internet. First Monday, 9, (1). [en línea] Disponible en: http://www.firstmonday.org/issues/ issueg 1/huffaker/ [consulta 2007, $11 \mathrm{de}$ mayo].

Jorge, C. (2002). Los indicadores de rendimiento como guía de la gestión: Aportaciones del Balanced Scorecard a las bibliotecas. Forinfa, 17 (julioseptiembre). [en línea] Disponible en: http://lemi.uc3m.es/forinf@/index. $\mathrm{php} /$ Forinfa/article/view/76 [consulta 2007, 12 de mayo].

Kajder, S.; Bull, G. (2003). Scaffolding for Struggling Students: Reading and Writing with Blogs. Learning\&Leading with Technology, 31, 2, 32-35.

L'Allier, J. J. Frame of Refrence: NETg's Map to the Products Their Structure and Core Belief. [en línea] Disponible en: http:// www.netg.com/research/whitepapers/ frameref.asp [consulta 2007, 10 de mayo].

Le Boterf, G. (2000). Construire les competentes individuelles et collectives. París: Editions d'Organisation.

Lévy-Levoyer, C. (2003). Gestión de las competencias. Barcelona: Gestión 2000 S. A.

Marqués Graells, P. (2007). Los medios didácticos. [en línea] Disponible en: http://dewey.uab.es/pmarques/medios. htm [consulta 2007, 10 de mayo].

Martínez Normand, L. (2001). Valoración de la accesibilidad y usabilidad de las páginas web españolas. VI Jornadas SIDAR 2001.[en línea] Disponible en: http://www.sidar.org/acti/jorna/ 6jorna/ponen6/loic/ [consulta 2007, 9 de mayo].

Martínez, D.; Martí, R. (2003). La factoría de recursos docentes. En: Los Centros de Recursos del Aprendizaje y la Investigación en los procesos de innovación docente: Jornadas Rebiun 2003. [en línea] Disponible en: http:// biblioteca.uam.es/paginas/palma.html [consulta 2007, 2 de mayo].

Millar, R. H. (2002). E-learning site usability checklist. [en línea] Disponible en: http://www.stcsig.org/usability/ resources/toolkit/e-learning-checklist. doc2002 [consulta 2007, 9 de mayo].

OCDE (2005). Definition and Selection of Competencies: Theoretical 
and Conceptual Foundations. Executive Summary [en línea]. Disponible en: http://www.oecd.org/ dataoecd/47/61/35070367.pdf [consulta 2007, 10 de mayo].

Placita, F. (2005). Aplicaciones de los weblogs para la enseñanza en línea. [en línea] Disponible en: http://www.hets. org/vp es weblogs.php [consulta 2007, 11 de mayo].

REBIUN (2006). IV Jornadas CRAI. Universidad de Burgos. [en línea]
Disponible en: http://www.ubu.es/ biblioteca/crai// [consulta 2007, 11 de mayo].

University of South Florida (2004). Directory of Online Resources for Information Literacy: Definitions of Information Literacy and Related Terms. [en línea]. Disponible en: http://bulldogs.tlu.edu/ mdibble/doril/definitions.html[consulta 2007, 9 de mayo].

\title{
PERFIL ACADÉMICO Y PROFESIONAL DEL AUTOR
}

Miguel Ángel Marzal. Profesor Titular de Universidad desde el curso académico 2001, pasando también a coordinar la asignatura Lenguajes Documentales. Durante los cursos 2003-04 y 2004-05 ha sido responsable de grupo de la asignatura Análisis de Contenido de la Licenciatura en Documentación. Director del Programa de Doctorado en Documentación de la Universidad Carlos III de Madrid, desde el 29 de enero de 2001. Este programa de Doctorado obtuvo la primera Mención de Calidad otorgada a un Doctorado en Documentación, en el año 2003, y una mención honorífica en la convocatoria de la Consejería de Educación de la Comunidad de Madrid de los I Premios a la mejora de la Calidad en las Universidades de la Comunidad de Madrid. La Mención de Calidad ha sido renovada en el año 2004. Imparte el curso Análisis Documental de Contenido y organización del conocimiento en Educación en este programa de Doctorado en Documentación.

E-mail:_mmarzal@bib.uc3m.es

DIRECCIÓN DEL AUTOR:

\author{
Miguel Ángel Marzal \\ Universidad Carlos III de Madrid (España) \\ c/ Madrid, 128 \\ 28903 Getafe-Madrid-España
}

Fecha de recepción del artículo: 17/10/07

Fecha de aceptación del artículo: 09/01/08 